\title{
Onlife-Lehre in Corona-Zeiten?
}

\section{Reflexionen über eine hochschulübergreifende Ringvorlesung zum thematischen Dreieck musikkulturelle Bildung - Digitali- sierung - Inklusion}

\author{
Frank Pätzold, Ina Henning und Imke Niediek
}

\section{Zusammenfassung des Beitrags}

Der vorliegende Beitrag befasst sich mit Potentialen eines neuen Lehr-Lernformats für lehramtsbezogene Studiengänge in Form einer hochschulübergreifenden Ringvorlesung, die zwischen analogen und digitalen Formaten alterniert. Die inhaltliche Konzeption der Ringvorlesung bewegt sich im Themenfeld musikkulturelle Bildung - Digitalisierung - Inklusion. Erste Auswertungen von Erfahrungen lassen Momente entlang der Zusammenhänge des Onlife oder Postdigitalen erkennen. Deutlich wird aber auch, dass ein gemeinsamer konjunktiver Erfahrungsraum durch die Verflechtung von digitalen und analogen Anteilen im Jetzt und in der Zukunft im Sinne einer Bildungspraxis immer wieder neu konstituiert und ausgehandelt werden muss.

\section{Schlüsselbegriffe: Hochschullebre $\bullet$ Digitalisierung $\bullet$ Onlife $\bullet$ Diskussionsbeitrag $\bullet$ Projektreflexion}

\section{Einführung}

Insbesondere für künstlerische bzw. musische Fächer und die Lehramtsstudiengänge stellt die pandemiebedingte Ad-hoc-Umstellung auf die Online-Lehre einen Verlust dar, der bislang nur unzureichend durch digitale Angebote kompensiert werden konnte. In einer aktuellen Befragung der Hochschule für Musik und Theater Rostock gaben 73 \% (bzw. 81\%)1 der Lehrenden von vier norddeutschen Musikhochschulen an, sie empfänden den fehlenden unmittelbaren Umgang mit den Studierenden als große Belastung, und 63 \% der Studierenden bestätigten, dass die fehlenden persönlichen Kontakte zu Lehrenden und Kommiliton*innen auch für sie eine große Belastung darstelle. Lediglich $2 \%$ der künstlerisch Lehrenden erachten digitale Lehrformate als vollwertige Ersatzform zum herkömmlichen Präsenzunterricht; 41 \% (bzw. $28 \%$ ) sehen darin zumindest noch eine praktikable Ergänzungsform, wohingegen $16 \%$ (bzw. $22 \%)$ der künstlerisch Lehrenden digitale Formate als gänzlich ungeeignete Vermittlungsform im Rahmen des künstlerischen Studiums ablehnen (vgl. Krämer/Hammerich 2021).

\footnotetext{
${ }^{1}$ Die Befragung wurde zweimal durchgeführt, drei Wochen nach dem verschobenen Semesterbeginn $2020(\mathrm{n}=327)$ und ein weiteres Mal zum Ende des Sommersemesters $2021(n=233)$.
} 
In dem Beitrag werden daher Potentiale der aktuellen Situation für neue Lehr-Lernformate diskutiert, die bewusst zwischen analogen und digitalen Formaten changieren. Grundlage des Beitrags sind erste Erfahrungen und informelle Rückmeldungen von Studierenden und Referent*innen einer interdisziplinären Ringvorlesung zum Thema Inklusion in der musikkulturellen Bildung: vom Analogen zum Digitalen ${ }^{2}$. Das Projekt stellt ein hochschulübergreifendes Studienangebot für Studierende von lehramtsbezogenen Studiengängen im Fach Musikpädagogik der Hochschule für Musik, Theater und Medien Hannover und Studierenden des Lehramts für Sonderpädagogik an der Leibniz Universität Hannover dar. Zielsetzung dieser Kooperation ist es, den Studierenden die Potentiale eines breiten Diversity-Verständnisses für die musikkulturelle Bildung sichtbar zu machen. Dabei wird den Studierenden eine kritische Auseinandersetzung mit Begriffen wie Talent, Befähigung und Behinderung ermöglicht, indem Künstler*innen und Pädagog*innen aus der musikkulturellen Praxis sowie Wissenschaftler*innen ihre Perspektiven auf das Themendreieck Inklusion, Digitalisierung und musikkulturelle Bildung darstellen.

\section{Vorstellung des Projekts}

Das Projekt ist zu Beginn der „zweiten Welle“ der Corona-Pandemie im November 2021 entstanden, als einerseits absehbar wurde, dass eine Rückkehr in den Präsenzbetrieb für längere Zeit nicht möglich sein würde. Andererseits wurde deutlich, dass die vollständige und plötzliche Umstellung auf eine reine Online-Lehre trotz eines massiven Ausbaus digitaler Infrastrukturen an beiden Hochschulen insbesondere die kreativen und sozialen Aspekte der beteiligten Studiengänge einschneidend beschränkte. Die Erfahrungen aus dem ersten und dem Beginn des zweiten Online-Semesters hatten den Bedarf an kooperativen Lernformaten gezeigt. Während Breakout-Sessions in Videokonferenzen und in Clouds gemeinsam bearbeitete Texte oder Präsentationen von Lehrenden wie Studierenden inzwischen weitgehend kompetent genutzt werden konnten, wurde ein Mangel an Angeboten deutlich, die die Studierenden neben wissensorientierten Aspekten des Lernens im Sinne einer „Transformation von Selbst- und Weltverhältnissen“ (Koller 2012, S. 15f.) auch auf kognitiver, emotionaler, sozialer und auch physisch-körperlicher Ebene anspricht. Die Universität als Lebensmilieu stellte keinen erfahrund begehbaren Raum mehr dar, Seminare wurden durch asynchrone Lernformate zeitlich und räumlich entgrenzt und die Studierenden in ihren Arbeitsprozessen häufig auf sich selbst zurückgeworfen. Dies galt insbesondere für die Studieneingangsphase, in der so gut wie keine der üblichen Sozialisations- und Initiationsrituale für tragfähige soziale Beziehungen sorgen konnten, die nun als Lerngruppen akademische oder auch soziale Unterstützung bieten können (vgl. Marczuk/Multrus/Lörz 2021).

Das Projekt sollte daher ein Angebot darstellen, das die Studierenden zu selbständiger Auseinandersetzung auf kognitiver, emotionaler, sozialer und auch physisch-körperlicher Ebene anregt und insofern nicht konventionelle und digitale Lernformate gegeneinander ausspielt

\footnotetext{
${ }^{2}$ Zum Zeitpunkt der Einreichung des Manuskripts war die Ringvorlesung noch nicht abgeschlossen, deshalb stellt der Beitrag eine erste Reflexion im Arbeitsprozess dar. Eine weitergehende Auswertung der Erfahrungen ist im Anschluss vorgesehen.
} 
oder das eine zur „schlechteren Notlösung“ für das andere werden lässt, sondern im Sinne Floridis Beschreibung des postdigitalen Zeitalters ein hybrides Modell entstehen lässt: „In Wahrheit sind wir weder on- noch offline, sondern onlife: Wir leben zunehmend in diesem besonderen Raum, der sowohl analog als auch digital, sowohl online als auch offline ist“ (Floridi 2017, S. 295).

Das Konzept der Ringvorlesung lässt sich folgendermaßen beschreiben: Im Sinne eines Scientific Walk treffen sich Studierende der beiden Hochschulen in Zweiergruppen einmal wöchentlich zu einem Spaziergang durch eine Parkanlage, der Eilenriede. Auf ihrem Spaziergang lernen die Tandems an fünf digitalen Impuls-Stationen verschiedene Perspektiven auf Inklusion in der musikkulturellen Bildung kennen, die von Referent*innen aus Wissenschaft, Kulturszene (also z.B. mixed-abled Tanzcompanies, Literaturprojekte, App-Musik-Projekte) und aus der inner- bzw. außerschulischen Bildungspraxis entwickelt wurden. Die Referent*innen wurden aufgrund ihres fachlichen Bezugs zu mindestens zwei der drei Kernbegriffe der Vorlesung - Inklusion, Digitalisierung oder musikkulturelle Bildung - für die Gestaltung eines Walks angefragt. Sie waren sowohl frei in der thematischen Schwerpunktsetzung oder Fragestellung ihres Impulses wie auch in der Gestaltung von Aufgabenformaten sowie der material-medialen Umsetzung (Fotos, kreative Aufgaben, Impulsfragen, Texte, Videos, Apps usw.). Die Impulse sollten geeignet sein, die Studierenden in ihrer inhaltlichen Auseinandersetzung akademisch oder künstlerisch anzuregen. Zur Umsetzung der Impulse werden verschiedene Medien wie die Smartphones der Studierenden und ein mobiles, digitales Whiteboard eingesetzt. Auf Wunsch einzelner Referent*innen wird an einigen Abenden im Anschluss an einen Walk eine gemeinsame Reflexion der Studierenden und Referent*innen per Videokonferenz angeboten. Die Studierenden führen im Seminarverlauf im Tandem ein E-Portfolio, das am Ende mit einer Reflexionsaufgabe als Studienleistung angerechnet werden kann.

\section{Erfahrungen}

Wir knüpfen in unseren ersten Überlegungen zur Reflexion von Erfahrungen mit dem Veranstaltungsformat an die These von Jörissen und Unterberg (vgl. 2019) an, dass wir uns auf dem Weg in eine postdigitale Gesellschaft befinden, in der „[...] die digitalen Infrastrukturen soweit ausgebaut sind, dass die durch sie etablierten Praktiken im Alltag der breiten Masse relevant werden und auch auf nichtdigitale Praktiken zurückwirken“ (Jörissen/Unterberg 2019, S. 12). Entlang welcher Momente lassen sich in dem Projekt Aspekte der These des Postdigitalen erkennen oder auch als „noch nicht“ und somit als „Zukunftsmusik“ beschreiben? Jörissen und Unterberg bieten für eine solche Analyse ein heuristisches Modell an, das die Zusammenhänge von Materialität, Sozialität, Medialität und Kulturalität zu beschreiben versucht. Demnach ist jede Kulturalität auf Medialität angewiesen, da keine Sozialität ohne Symbole gedacht werden kann - ergo keine Kulturalität ohne Medialität. Zum zweiten ist Medialität immer auch an eine materielle Grundlage gebunden - ergo keine Medialität ohne Materialität. Drittens ist Materialität wiederum an kulturelle Formen, Diskurse und soziale Praktiken gebunden - ergo keine Materialität ohne Kulturalität (vgl. Jörissen/Unterberg 2019, S. 12). Schließlich gehen wir aber auch umgekehrt davon aus, dass Medialität in sozialen Praktiken 
hervorgebracht wird. Insofern möchten wir in einem vierten Schritt dem Gedanken „Keine Medialität ohne kulturelle und soziale Formen“ nachspüren. Die leitende Fragestellung der folgenden Reflexionsmomente lautet: Inwieweit lassen sich in den Erfahrungen aus den ersten Walks Anzeichen solcher Wechselwirkungen erkennen, wie Jörissen und Unterberg sie für die Postdigitalität postulieren?

\section{Keine Kulturalität und keine Sozialität obne Medialität}

Bei der Vorlesung handelt es sich um eine Wahlpflichtveranstaltung, die in den Vorlesungsverzeichnissen der beteiligten Hochschulen beschrieben war. Bereits der Titel der Veranstaltung Inklusion in der musikkulturellen Bildung: vom Analogen zum Digitalen hat einen medialen Charakter und stellt einen Rahmen her, in dem sich die Veranstaltung bewegen soll: Dabei bleibt bewusst unbestimmt, ob sich der Titel ausschließlich auf das Veranstaltungsthema oder auch auf methodische und mediale Aspekte der Veranstaltung selbst bezieht. Die Wege- oder Prozessmetapher verweist zudem auf eine zu erwartende Dynamik, die hier zwar als Spannungsbogen der Veranstaltung erscheint. Tatsächlich beschreibt sie aber auch eine ganz konkrete Anforderung an die Studierenden, sich kognitiv, motorisch und emotional-sozial in Bewegung zu bringen. Hier lässt sich auch mit Jörissen und Unterberg argumentieren, dass in ästhetischen Prozessen und Vollzügen, die die Ringvorlesung adressiert, das Potential liegt, Digitalität umfassender zu erfahren, als dies mit bloßen kognitiven Mitteln möglich ist (vgl. Jörissen/Unterberg 2019). Die Fokussierung auf die Rahmenbegriffe Inklusion, Digitalisierung und musikkulturelle Bildung erweist sich als ein von den Referent*innen flexibel genutztes Feld, das die Vielfalt der Kulturellen Bildung als ästhetisch konstitutives Element betrachtet. Die Vielfältigkeit der Umsetzung der Impulse der Referent*innen in direkten oder indirekten Bezügen zu den Schnittfeldern ruft Antworten der Studierenden hervor, die zu pluralem bzw. vielschichtigem Denken, kreativen Äußerungen und performativen Akten in verstärktem Maße ermutigen sollen. Auf diese Weise bereitet der Titel den Boden für die Entwicklung der Seminarkultur.

Auch der Veranstaltungsort selbst hat eine symbolische Funktion. Bewusst wurde einerseits ein öffentlicher Ort gewählt, der aber nicht großstädtisch anmutet und typischerweise mit dem Label digital in einen Zusammenhang gebracht wird, sondern für Natur und sinnlich-körperliche Erfahrungen steht. Dahinter steht die Überlegung, dass ein schwieriges oder unbekanntes Thema als Terrain auch neu vermessen werden muss und es daher sinnvoll sein kann, auch die äußerlichen Bedingungen der Aneignung zu verändern (zu innovativen Maßnahmen zur Lernraumgestaltung als hochschulweite Strategie vgl. Ninnemann/Jahnke 2018).

Weitere Elemente vermitteln Grundgedanken der Seminarkonzeption, die zu einer gelebten kulturellen Praxis werden soll: Die bewusste Irritation von üblichen universitären Lehrformaten und die thematische Vielfalt der Impulse soll einerseits dazu beitragen, bei den Studierenden innere Bereitschaft und Neugier zu wecken, sich mit einer Frage oder einer Idee der Referent*innen auseinanderzusetzen. Die Arbeit in einem Tandem über das gesamte Semester hinweg und der Ritualcharakter der Walks soll andererseits sowohl Stabilität und Sicherheit bieten als auch resonante Beziehungen und unmittelbares Feedback in der Auseinandersetzung mit den unterschiedlichen Impuls-Beiträgen ermöglichen. Verschiedene Angebote wie 
z.B. der wöchentliche Infobrief per Mail zu den einzelnen Walks, die Abendgespräche mit den Referent*innen und Online-Präsenztermine zur Vor- und Nachbereitung der Veranstaltung sollen einen sozialen Raum eröffnen, der bei aller Unbestimmtheit die symbolische Einheit einer Vorlesung als „Lern-Gemeinschaft“ aufrechterhält. Im Sinne von Jörissen und Unterberg wird das komplexe Phänomen der Digitalität auch hier aus dem engen technikzentrierten Fokus medialer Phänomene herausgelöst (vgl. 2019, S. 14), indem zwar eine thematische Fokussierung (Digitalität als Thema und als Metaphänomen) gegeben ist; tatsächlich aber öffnet die Medialität in unserem Verständnis Räume, die sich sozial und kulturell erst von Menschenhand formieren müssen.

\section{Keine Medialität obne Materialität}

Die Vorlesung sollte zwar bewusst eine Verbindung zwischen digitalen und analogen Elementen herstellen, dennoch war die Vehemenz, mit der die material-physischen Bedingungen den Verlauf der Vorlesung beeinflusst haben, bemerkenswert. Die Vorlesung fand im dritten Online-Semester der Pandemie statt. Dennoch stellte sich zu Beginn heraus, dass nicht alle Studierenden die materiell-technischen Voraussetzungen mitbringen konnten, um beispielsweise filmische Impulse durch ihre mobilen Endgeräte im öffentlichen Raum zu streamen. Hier wurden einerseits Einzelfalllösungen gefunden, andererseits wurden datenintensive Impulse den Studierenden vorab zur Verfügung gestellt und erst auf dem Walk mit einer Aufgabe oder Fragestellung ergänzt. Die Impulse mussten mit den Ressourcen der verschiedenen Betriebssysteme, Speicherkapazitäten, Mobilfunkverträge der Endgeräte der Studierenden abgestimmt werden. Das Installieren von bestimmten Apps für einen einzelnen Walk bedeutete zudem zusätzliche Vorbereitungszeit für die Studierenden, die über das dafür notwendige technische Know-how verfügen müssen und sich mit den datenschutzrechtlichen Bedingungen der AppBetreiber einverstanden erklären müssen, um dieses Angebot nutzen zu können.

Insbesondere in pädagogischen Zusammenhängen spielen Dinge als Vermittler gesellschaftlicher Wissensbestände und Medien für die Auseinandersetzung mit sich und der Welt eine besondere Rolle (vgl. Nohl 2011). Hier bestätigt sich Jörissen und Unterbergs These, dass Code und Software mittlerweile nicht nur digitale Räume strukturieren, sondern ihre konstitutive Kraft auch im materiellen Raum entfalten können (vgl. 2019, S. 14). So wurde das Waldstück mit digitalen und materialen Medien ergänzt und verändert bzw. eingerichtet: An den betreffenden Stationen hingen QR-Codes, die unter anderem zu Filmen und Texten führten. Darüber hinaus stand ein digitales Whiteboard (mit mobiler Stromquelle) für das kooperative Arbeiten an einer Station sowie Beutel oder Kisten mit ergänzenden Materialien bei den verschiedenen Walks zur Verfügung.

Die Diffusion von online und offline (im weitesten Sinn also Momente des Onlife) durch die Verbindung von räumlich-materiellen und „virtuellen“ bzw. digitalen Aspekten zeigte sich in der Bewältigung der Aufgabenstellungen der Referent*innen für die Studierenden: Eine Aufgabenstellung sah beispielsweise vor, dass die Studierenden analog auf vorbereiteten Karteikarten einen Ort im Stadtwald kartografieren und zusätzlich zu den materiellen Gegebenheiten auch die unterschiedlichen Geräusche in diesem Lageplan abbilden. Die so entstandenen Karten wurden zum Abschluss für die Rückmeldung an die Referent*innen in 
an Bäumen aufgehängte Beutel gelegt. Um die individuellen Lagepläne auch für die eigene Lerndokumentation zu erhalten, wurden diese von den Studierenden wie selbstverständlich digitalisiert, abfotografiert. Andere Beiträge erscheinen insofern onlife, als sie unabhängig von einer konkreten, materiellen Umgebung wie dem Wald-Spaziergang, einem Hörsaal oder einem Videokonferenztool von den Studierenden erlebt werden konnten: Die geforderte Auseinandersetzung der Studierenden mit der Lebenswirklichkeit von Menschen mit Down-Syndrom beinhaltete verschiedenste Aufgabenstellungen, die keine explizite Nutzung des Raumes vorsahen. Die Studierenden brachten ihre (emotionale) Betroffenheit mit der Lebenswirklichkeit der Referent*innen mit Behinderungserfahrung spontan in der Eilenriede durch digitale Audiobeiträge zum Ausdruck, die sie später in ihre digitalen Lerntagebücher integrierten.

Die Studierenden erleben eine Abhängigkeit des Lernens von den räumlich-materiellen Bedingungen in neuer Weise: Im Wald bin ich als Materie ganz anders gefordert, es entsteht eine Körperlichkeit in Differenz zur Körperlichkeit der Online-Lehre. Sie wird mehrsinnlich erfahren, die Positionierung von Körpern im Raum und die Wahrnehmung dieser Räumlichkeit verschieben sich im Vergleich zur klassischen Präsenzlehre, aber auch zur Online-Lehre. Erste Rückmeldungen zeigen beispielsweise, dass es mehrere Wochen brauchte, um eine Wegeroutine so zu etablieren, dass das Finden des richtigen Wegs weniger Aufmerksamkeit und Energie benötigte und sich die Studierenden auf die inhaltliche Arbeit konzentrieren konnten.

\section{Keine Materialität außerhalb der kulturellen Formen}

Das Format einer Lehrveranstaltung im öffentlichen Raum zeigt eine bislang kaum wahrnehmbare Komponente der Beziehung zwischen Materialität und kulturellen Formen in der Hochschullehre: In der zweiten Veranstaltungswoche wurde das Waldstück für den öffentlichen Besucherverkehr geschlossen, da die zuständige Behörde aufgrund einer Sturmwarnung kein Risiko eingehen wollte. Mit der Konfrontation mit verschiedenen Wetterlagen hatte das Vorbereitungsteam gerechnet, nicht aber mit einem „Ausfall“ der Örtlichkeit.

Vor dem Hintergrund der Auflösung räumlich-materieller und zeitlicher Gewissheiten, die ein Studium vor der Corona-Pandemie ausgemacht hat, wird auch in dieser Vorlesung sichtbar, wie sehr die kulturellen Formen des Studierens in materielle Gegebenheiten eingelagert sind: Das regelmäßige Aufsuchen von Gebäuden und Räumen wurde abgelöst von der Notwendigkeit, sich mehr oder weniger kompetent in verschiedenen Lernmanagement- und Videokonferenzsystemen zu bewegen. Asynchrone Online-Vorlesungen sowie Selbststudienund Gruppenaufgaben dominieren den Studienalltag statt wöchentlicher Stundenpläne im 90-Minuten-Takt. Das Wegbrechen solcher gewohnten Strukturen und Routinen, die sich im Umgang mit Räumen und Dingen zeigen, führt bei den Studierenden zu einem Verlust von Gewissheit und zu Verunsicherung.

Gleichzeitig haben sich nach zwei reinen Online-Semestern neue kulturelle Formen des Studierens mit eigenen materialen Qualitäten etabliert: Aufgrund der oben bereits beschriebenen Sturmwarnung wurde die Veranstaltung in der zweiten Veranstaltungswoche vollständig in den „virtuellen Raum“ des Lernmanagementsystems einer der beiden Hochschulen verlegt, so dass dieser Termin online absolviert werden musste. Einige Studierende fragten daraufhin, ob nicht künftig alle Veranstaltungen ausschließlich online stattfinden könnten (oder die Walks 
auch mal in ihrem Stadtteil stattfinden könnten), da es doch aufwändig sei, sich jede Woche auf eine mögliche Covid-19-Infektion testen zu lassen, um dann im Stadtwald eine Lehrveranstaltung zu besuchen. Vor dem Hintergrund der Erfahrungen mit den physisch-körperlich weit weniger aufwändigen Veranstaltungen der Online-Lehre der vergangenen zwei Semester deuten wir diese Rückmeldung so, dass sich einerseits eine neue kulturelle Form etabliert hatte, die den Besuch einer Vorlesung vom heimischen Schreib- oder Küchentisch aus zum Normalfall werden lässt. Andererseits hatte sich (noch) kein konjunktiver Erfahrungsraum bzw. eine gemeinsame kulturelle Form entwickeln können, in der das Aufsuchen des Waldgebiets zum inhärenten Bestandteil der Vorlesung geworden ist.

Am Ende des Vorlesungszeitraums lässt sich aber rückblickend feststellen, dass die Veranstaltung im Vergleich nur sehr geringe Fehlzeiten von Studierenden aufweist, sodass wir dies als Hinweis interpretieren, dass sich eine solche Form inzwischen etablieren konnte.

\section{Keine Medialität obne kulturelle und soziale Formen}

Auch die Referierenden haben verschiedene Herausforderungen in der Gestaltung ihrer Impulse erlebt: Jörissen und Unterbergs Aussage, Digitalität manifestiere sich nicht nur informationell-technisch, sondern gleichermaßen ästhetisch-kulturell (vgl. 2019, S. 19), bewahrheitete sich in besonderer Weise: Manche Referierende konnten tatsächlich nur über das Betreten eines stellvertretenden Raums - eines „Alternativwalds“, in dem sie sich filmten - die für sie erforderliche ästhetische Qualität für einen Video-Impuls, der im Stadtwald per QR-Code zugänglich gemacht wurde, generieren. Für einige stellte es hingegen eine Herausforderung dar, Impulse zu entwickeln, die ohne direkte Feedbackmöglichkeit und Interaktion mit den Studierenden entstehen.

Die Einführung der Online-Auswertungsgespräche am Abend einzelner Walks sind auf dieses Bedürfnis nach Feedback, aber gegebenenfalls auch auf Verunsicherung zurückzuführen, die ohne eine Resonanz auf einen erarbeiteten Impuls entsteht. Für Referent*innen, die nicht aus Bildungskontexten im weitesten Sinne kommen, scheint die Perspektivenübernahme für die Frage, was Studierende brauchen, um einen Impuls gut nutzen zu können, besonders schwierig. Da es keine gemeinsamen Erfahrungsräume gibt, die zur Vorbereitung angerufen werden können und in den Situationen kein direkter Kontakt vorhanden ist, um spontan nachzujustieren, stellen die Impulse fragile bzw. riskante Konstrukte dar, deren Aneignung sich jeder Kontrolle entzieht. Zugleich widerspricht es dem Konzept und den Inhalten der Veranstaltung, kleinschrittige und eindeutige Arbeitsaufträge $\mathrm{zu}$ formulieren, wie es in anderen asynchronen Vorlesungen mit Selbststudienaufgaben zur bloßen Wissensabfrage der Fall ist. Erste Rückmeldungen und Fragen von Studierenden weisen darauf hin, wie stark der Bruch der Veranstaltung mit der klassischen Vorlesung tatsächlich ist: Studierende erleben, dass Lehrende weniger greifbar bzw. wahrnehmbar sind und hinter den digitalen Angeboten zurücktreten.

Die Vorstellung, dass Lehrende oder Referierende einen Wissensvorsprung haben und die Veranstaltung ein Ort des Wissenstransfers ist, führt zu massiver Verunsicherung, da die Intention der Vorlesung bewusst mit der Vorstellung des „Nürnberger Trichters“ bricht und vielmehr ein Angebot darstellen will, die Selbst- und Weltverhältnisse zu hinterfragen und 
weiterzuentwickeln. Daher kann die wiederkehrende Frage von Studierenden, ob eine Übung oder Frage „richtig“ bearbeitet wurde, nicht eindeutig beantwortet werden.

Sich eigenständig Inhalte auf einem Spaziergang zu erschließen und dabei die alleinige Zuständigkeit sowohl für die inhaltliche Aneignung der Impulse wie auch für die technische Herstellung selbst zu tragen, wird von einigen Studierenden als sehr herausfordernd erlebt (zum Vergleich: Bei einer Online-Vorlesung durch ein Videokonferenzsystem gibt es zwei bzw. drei Seiten, die konstitutiv für diese Art der Vorlesung sind und damit zum Gelingen beitragen: technische Infrastruktur der Hochschule, technische Ressourcen und Kompetenzen der Lehrenden, technische Ressourcen und Kompetenzen der Studierenden).

Das Format macht daher unterschiedliche Herangehensweisen von Studierenden im Vergleich zur konventionellen Präsenzlehre deutlicher sichtbar: So gibt es Studierende, welche die erforderliche Selbstorganisation weitgehend eigenständig bewältigen und eigene Entscheidungen lediglich mit kurzen Rückfragen absichern. Andere erleben erst durch kleinschrittige Orientierungshilfen und ständige Bestätigung genügend Rückhalt, um sich im wahrsten Sinn des Wortes jede Woche erneut „auf den Weg machen“ zu können. Im Verlauf der Vorlesung zeigte sich, dass es einen Lernprozess darstellt, mit derartigen Anforderungen umgehen zu lernen und - auf sich selbst verwiesen - Bildungsprozesse tatsächlich als aktive Aneignung zu verstehen, die sich insbesondere in der Hochschullehre nicht in der Differenz zwischen Educant $^{*}$ in und Educator*in erschöpft, sondern nur begrenzt der Steuerung und Kontrolle von Lehrenden unterliegen kann.

Die kontinuierliche Tandemarbeit sollte es ermöglichen, Formen der Zusammenarbeit einzuüben und Lernen als Prozess der Erkenntnisbildung zu verstehen. Die Portfolios sollten schließlich auf einer weitergehenden Reflexionsebene die Arbeit an Fragestellungen ermöglichen, die über eine einfache Dokumentation des Erlebten hinausreichen. Tatsächlich zeigten sich in den letzten Wochen der Walks erste Momente, die auf einen solchen Lernprozess hindeuten: Studierende fragen als Gruppe nach einer gemeinsamen Hausarbeit, die klassischerweise als Einzel- oder maximal Tandemarbeit ausgelegt ist, weil sie an einem Problem aus mehreren Perspektiven gemeinsam arbeiten wollen. Andere formulieren Fragen in ihren Portfolios, die darauf verweisen, dass es um mehr als nur um einen verstehenden Nachvollzug der Inhalte geht, sondern eigene Problem- und Fragestellungen aus den Impulsen der Referent*innen entstehen und diese so weitergedacht- und entwickelt werden.

\section{Ausblick}

Für die Auswertung der Projektarbeit nach Abschluss der Vorlesungszeit (vorgesehen ist die Auswertung der E-Portfolios der Studierendentandems sowie Interviews mit Studierenden und Referierenden) sollen die hier skizzierten Reflexionsmomente einen Zugang ermöglichen: Wie haben sich die Studierenden die virtuellen und materiellen Räume, die die Ringvorlesung bietet, selbsttätig angeeignet (vgl. Deinet/Reutlinger 2014)? Welche Materialitäten des Ortes wurden wie genutzt, wo haben sie gesessen/gestanden, welche Fragestellungen haben sie im Gehen bearbeitet? Welche Effekte hat das Arbeiten im öffentlichen Raum, beispielsweise durch die Begegnung mit oder Beobachtung durch Passant*innen im Vergleich zur Arbeit im Hörsaal oder im digitalen Videokonferenzraum? 
Übergeordnet für die weitergehende Auswertung der Erfahrungen mit den Online-Semestern stellt sich uns aber die Frage: Wie viel Irritation verträgt Lehre in Zeiten, in denen Universität nicht mehr sichtbar bzw. greifbar ist, in denen kein konkreter Ort, keine konkrete Materialität mehr strukturgebende Momente darstellen, die für Orientierung und psychologische Sicherheit sorgen, um sich auf den Inhalt einlassen zu können? Darüber hinaus beschäftigt uns die Beobachtung, dass zumindest in formellen Bildungszusammenhängen (wie einem Hochschulstudium) materielles, kulturelles und soziales Kapital der Studierenden auch unter Ausrufung eines postdigitalen Zeitalters wirkmächtige Einflussfaktoren sind. Wir fragen uns: Wie haben die Studierenden diese Herausforderungen erlebt?

Zusammenfassend lässt sich allerdings schon jetzt feststellen, dass es gelungen ist, Momente des Postdigitalen herzustellen, in denen die Materialität oder Virtualität von Räumen keine Relevanz hat und in denen sich Praktiken etablieren, die daher als onlife bezeichnet werden können. Deutlich wurde aber auch, dass es eben keinen dem Projekt vorgängigen gemeinsamen konjunktiven Erfahrungsraum im Sinne Nohls (2011) gab, auf den alle Beteiligten unausgesprochen zugreifen konnten. Vielmehr zeichnet sich das Projekt durch fortwährende Herstellungs- und Verständigungsarbeit aus, die im Laufe der Vorlesungswochen nach und nach eine gemeinsame Praxis entstehen lässt, die im weitesten Sinn als onlife bezeichnet werden kann. Damit stellt das Projekt eine Bildungspraxis par excellence dar: Auch in einer künftigen Durchführung wird es erneut zentrale Aufgabe sein, diese Herstellungsarbeit zu leisten und mit neuen Referierenden und Studierenden einen konjunktiven Erfahrungsraum für Bildungsprozesse zu schaffen.

\section{Literatur}

Deinet, Ulrich/Reutlinger, Christian (2014): Tätigkeit - Aneignung - Bildung. Einleitende Rahmungen. In: Deinet, Ulrich/Reutlinger, Christian (Hrsg.): Tätigkeit - Aneignung - Bildung. Positionierungen zwischen Virtualität und Gegenständlichkeit. Wiesbaden: Springer VS, S. 11-30.

Floridi, Luciano (2017): Die Mangroven-Gesellschaft. Die Infosphäre mit künstlichen Akteuren teilen. In: Otto, Philipp/Gräf, Eike (Hrsg.): 3Th1CS - Die Ethik der digitalen Zeit. Berlin: iRights.Media.

Jörissen, Benjamin/Unterberg, Lisa (2019): DiKuBi-Meta [TP1]: Digitalität und Kulturelle Bildung. Ein Angebot zur Orientierung. In: Jörissen, Benjamin/Kröner, Stephan/Unterberg, Lisa (Hrsg.): Forschung zur Digitalisierung in der Kulturellen Bildung, S. 11-24. München: kopaed. URL: pe docs.de/volltexte/2020/18486/pdf/Joerissen_Kroener_Unterberg_2019_Forschung_zur_Digital isierung.pdf (18.06.2021).

Koller, Hans-Christoph (2012): Bildung anders denken. Einführung in die Theorie transformatorischer Bildungsprozesse, Stuttgart: Kohlhammer.

Krämer, Oliver/Hammerich, Helen (2021): Einstellungen von Lehrenden und Studierenden zur digitalen Lehre im Corona-Semester 2020. URL: hochschulforumdigitalisierung.de/de/blog/musikhochschulen-meinungsstreit (18.06.2021).

Marczuk, Anna/Multrus, Frank/Lörz, Markus (2021): Die Studiensituation in der Corona-Pandemie. Auswirkungen der Digitalisierung auf die Lern- und Kontaktsituation von Studierenden. In: DZHW Brief 01|2021. Hannover: DZHW. URL: doi.org/10.34878/2021.01.dzhw_brief. 
Ninnemann, Katja/Jahnke, Isa (2018): Den dritten Pädagogen neu denken - Wie CrossActionSpaces Perspektiven der Lernraumgestaltung verändern. In: Getto, Barbara/Hintze, Patrick/Kerres, Michael (Hrsg.): Digitalisierung und Hochschulentwicklung. Proceedings zur 26. Tagung der Gesellschaft für Medien in der Wissenschaft e.V. Münster/New York: Waxmann, S.135-147.

Nohl, Arnd-Michael (2011): Pädagogik der Dinge. Bad Heilbrunn: Klinkhardt. 


\section{Informationen zu den Autorinnen und zum Autor}

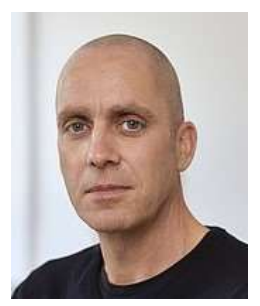

Frank Pätzold ist wissenschaftlicher Mitarbeiter am Institut für Sonderpädagogik in der Abteilung Pädagogik bei Beeinträchtigungen der geistigen Entwicklung an der Leibniz Universität Hannover. Seine Arbeitsschwerpunkte sind schulische Inklusion im Förderschwerpunkt geistige Entwicklung, sonderpädagogische Professionalisierungsprozesse und Digitalisierung in der Lehrer*innenbildung.

frank.paetzold@ifs.uni-hannover.de

www.ifs.uni-hannover.de/de

Ina Henning, Dr., hat die Juniorprofessur für Musikpädagogik mit dem Schwerpunkt Inklusion und Heterogenität an der Hochschule für Musik, Theater und Medien Hannover inne. Sie forscht im Bereich Inklusiver Musikpädagogik und -didaktik, in inklusiven Settings in der inner- und außerschulischen Kulturellen Bildung sowie zu künstlerischer Forschung in pädagogischen Kontexten.

ina.henning@hmtm-hannover.de

www.ifmpf.hmtm-hannover.de

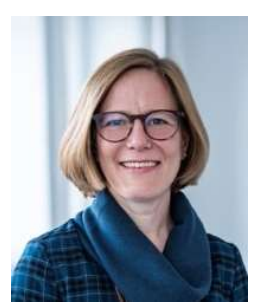

Imke Niediek, Dr., ist Professorin für Pädagogik bei Beeinträchtigungen der geistigen Entwicklung an der Leibniz Universität Hannover. Ihre Arbeitsschwerpunkte sind die Dispositive von Macht und Wissen im Kontext von Behinderung, Mechanismen von Teilhabe und Ausschluss in Interaktionssituationen, qualitative Forschungsmethoden im Kontext Behinderung sowie Unterstützte Kommunikation.

imke.niediek@ifs.uni-hannover.de

www.ifs.uni-hannover.de/de

\section{Zitationshinweis:}

Pätzold, Frank/Henning, Ina/Niediek, Imke (2021): Onlife-Lehre in Corona-Zeiten? Reflexionen über eine hochschulübergreifende Ringvorlesung zum thematischen Dreieck musikkulturelle Bildung - Digitalisierung - Inklusion. In: Online-Magazin Ludwigsburger Beiträge zur Medienpädagogik, Ausgabe 21/2021. URL: medienpaed-ludwigsburg.de 